\title{
What Ejected Nebulae around Evolved Massive Stars Tell us of the Rotation of their Central Star
}

\author{
Antonella Nota \\ Space Telescope Science Institute, 3700 San Martin Drive Baltimore \\ $M D$ 21218, and \\ European Space Agency
}

\begin{abstract}
Most LBVs and Ofpe/WN9 stars in the Milky Way and in the Magellanic Clouds are surrounded by associated circumstellar nebulae which have been ejected in some previous phase of their evolution. These nebulae are the fossil record of the interactions of previous winds and of the violent ejections in which the stars most likely have shed their outer layers. The study of the morphology, kinematics and chemical composition of the ejected material has allowed us to gain deep insight in the ejection and shaping mechanism, and in the properties of the central star at the moment of the ejection. In this review I will address how ejected nebulae are providing independent supporting evidence that rotation plays a major role in the evolution of massive stars.
\end{abstract}

\section{Introduction}

Massive stars (initial mass $M_{i} \geq 20-30 M_{\odot}$ ) spend their hydrogen-burning phase as $\mathrm{O}$ stars, and their helium-burning phase as Wolf-Rayet (WR) stars (e.g. Maeder \& Chiosi 1986). In the intermediate evolutionary stages, they experience extreme mass loss and/or giant eruptions, and the total amount of stellar wind material lost controls the subsequent evolution, namely whether an $\mathrm{O}$ star can evolve into a WR star or not. These intermediate phases are identified with emission-line objects such as Luminous Blue Variables (LBVs), Ofpe/WN9 and $\mathrm{B}[\mathrm{e}]$ stars, when $\mathrm{M}_{\mathrm{i}} \geq 50 \mathrm{M}_{\odot}$. Alternatively, $\mathrm{O}$ stars are expected to evolve through a red supergiant (RSG) stage prior to the WR phase if $20 \leq \mathrm{M}_{\mathrm{i}} \leq 50 \mathrm{M}_{\odot}$.

LBVs in particular are located close to the upper luminosity/stability limit in the H-R diagram (the Humphreys-Davidson (H-D) limit; Humphreys \& Davidson 1979) and are characterized by irregular photometric (0.5 - $2 \mathrm{mag})$ and spectral variations (Fig. 1). The Ofpe/WN9 stars (Walborn 1977) are believed to be closely related to LBVs in that they represent the hot, minimum state. The $\mathrm{B}[\mathrm{e}]$ stars occupy a similar position in the HR diagram to these stars but their evolutionary status is not well established. The relics of the extensive mass loss and/or giant eruptions which characterize the LBV transition phase are seen as circumstellar shells which typically have ionized masses of $1-4 \mathrm{M}_{\odot}$ and sizes of $0.2-2$ pc (e.g. Nota et al. 1995). The most famous of these relics is the Homunculus nebula associated with the LBV $\eta$ Car whose true bipolar structure has been studied in detail (Davidson \& Humphreys 1997; King et al. 2002).

These nebulae have provided very important clues on the evolutionary history of the central star. Their morphology has helped constraining the outburst mechanism. Their dynamical timescales have provided estimates to the duration 


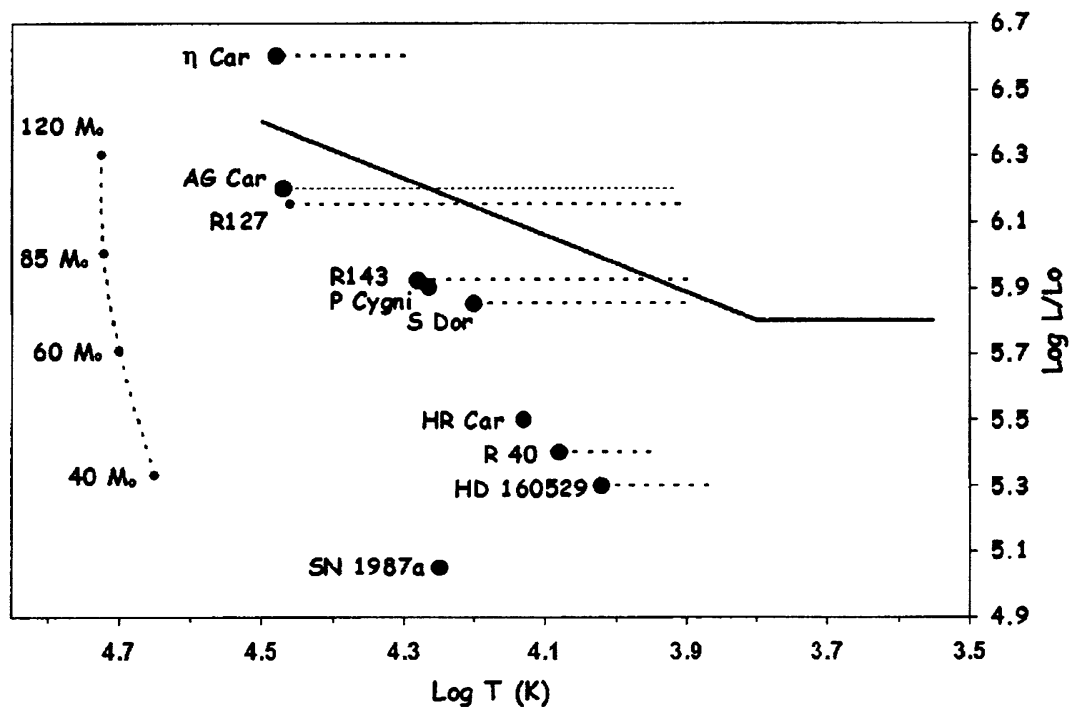

Figure 1. Upper H-R Diagram, adapted from Humphreys \& Davidson (1994). The upper luminosity Humphreys-Davidson limit is indicated, together with the locations of known LBVs.

of the transition phase. Since the nebulae, in effect, represent the stellar surface layers during the transition phase, the surface composition can be inferred by measuring the nebular abundances.

Ejected nebulae are an ideal laboratory to study the conditions prevailing in the star at the time of maximum mass loss, and I shall discuss in this review how they have provided independent evidence of the role of rotation in massive stars.

\section{Are All Evolved Massive Stars Surrounded by Ejected Nebulae?}

I shall concentrate for this review on LBVs and Ofpe/WN9 stars. The vast majority of LBVs and Ofpe/WN9 stars in our Galaxy and in the Magellanic Clouds are surrounded by ejected nebulae which are physically associated with the central star. These are the result of a combination of mass lost continuously over time and of violent ejections in which the star has possibly lost its outer layers (Table 1).

\section{The Nebular Observables}

Observations (direct imaging in optical/IR wavelengths, spectroscopy) of ejected nebulae have provided a wealth of information on their physical and chemical characteristics. These include 1) the morphology, 2) the kinematics, which yields the dynamical timescale, 3) the total flux, which yields the ejected mass 
Table 1. Frequency of ejected nebulae around LBVs, Ofpe/WN9 stars and candidates in the Milky Way and in the Magellanic Clouds

\begin{tabular}{|c|c|c|c|}
\hline \multicolumn{2}{|c|}{ Confirmed LBVs } & LBV Candidates & Ofpe/WN9 \\
\hline Milky Way & $\mathrm{LMC} / \mathrm{SMC}$ & Milky Way & LMC \\
\hline$\eta \operatorname{Car}^{(*)}$ & S Dor?(*) & WRA751(*) & BE294 \\
\hline P Cygni ${ }^{(*)}$ & $\mathrm{R} 71^{(*)}$ & $\mathrm{G} 79.29+0.46^{(*)}$ & S9 \\
\hline AG Carinae ${ }^{(*)}$ & $\mathrm{R} 127^{(*)}$ & $\mathrm{G} 25.5+02^{(*)}$ & R84 \\
\hline HR Carinae ${ }^{(*)}$ & HDE269582 & HD168625(*) & R99 \\
\hline HD160529 & R110 & He3-519 $(*)$ & $\mathrm{S} 61^{(*)}$ \\
\hline & $\mathrm{R}_{143^{(*)}}$ & & $\mathrm{BE} 381^{(*)}$ \\
\hline & $\mathrm{R} 4(\mathrm{SMC})^{(*)}$ & & $\mathrm{S} 119^{(*)}$ \\
\hline
\end{tabular}

$(*)$ indicates presence of an associated nebula

of ionized, neutral gas, and dust, 4) the temperature and the ionization characteristics, and 5) the chemical composition. Out of all these morphology and chemical composition do provide an independent indication that rotation has played an important role in the evolution of the star at the moment of the nebula formation.

\subsection{The Morphology}

LBV and Ofpe/WN9 associated nebulae display a continuous range of morphologies, from extremely bipolar to mildly bipolar to elliptical. In all cases there is clear evidence of a preferential symmetry axis.

The extremely bipolar nebulae (Fig. 1) are well represented by $\eta$ Carinae, where two expanding bubbles are attached at a narrow waist, and are aligned with a preferential axis (Davidson et al. 1997; Schulte-Ladbeck et al. 1988b; King et al. 2002). The nebulae around HR Carinae (Hutsemékers \& Van Drom 1991a; Clampin et al. 1995; Weis et el. 1997; Nota et al. 1997; Machado et al. 2002), G25.5+0.2 (Clark, Steele \& Langer 2000) and the LBV candidate HD168625 (Hutsemékers et al. 1994; Nota et al. 1997; Robberto \& Herbst 1998; Pasquali et al. 2002 ) are also bipolar, although in these two cases the morphology is less visually striking. Both HR Carinae and HD168625 nebulae are $\simeq 5000 \mathrm{yr}$ old. In the case of HR Carinae only the southern bubble is visible, and fragmented, and the kinematic data confirms presence of a truly bipolar outflow.

Most of the remaining known nebulae display much milder asymmetries (Fig. 2). AG Carinae (Smith et al. 1997; Trams et al. 1996; Schulte-Ladbeck et al. 1994; Leitherer et al. 1994), R127 (Walborn 1982; Stahl 1987; Clampin 1993; Smith et al. 1998), WRA751 (Hu et al. 1990; Hutsemékers \& van Drom 1991b; de Winter et al. 1992) and S119 (Nota et. al xxxx; Schulte-Ladbeck et al. 2003) are four representatives of the more conspicuous class of nebulae where a preferred axis is still present, with an overall symmetry that is elliptical. 

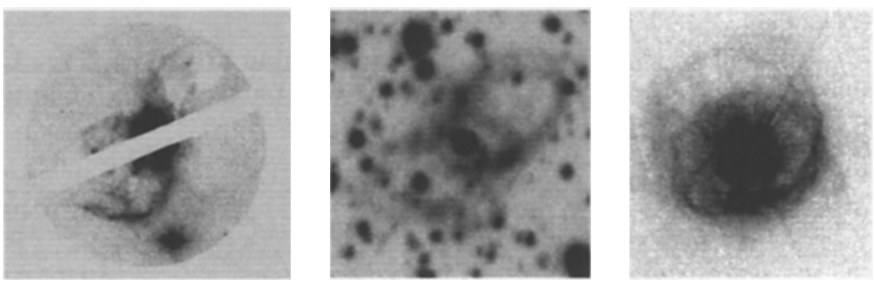

Figure 2. Four examples of galactic extreme bipolar nebulae, in the light of $H \alpha: \eta$ Car (courtesy of R. White), HR Car, G25.5+0.2, and HD168625. In all images, North is up and East to the left.
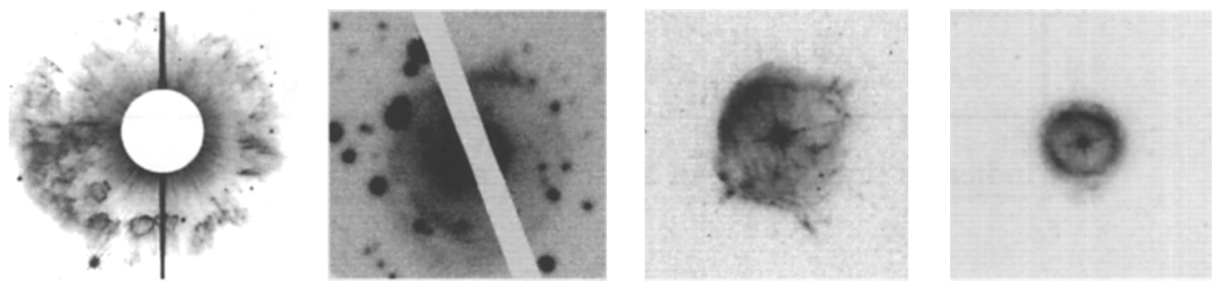

Figure 3. Four examples of elliptical nebulae in the light of $H \alpha$ : AG Carinae and WRA751 are galactic, S119 and S61 are in the LMC. In all images North is up and East to the left. The images are not equally scaled.

AG Carinae and R127 are remarkably similar: AG Carinae is galactic, $\simeq$ $36^{\prime \prime} \times 36^{\prime \prime}$ in size, while R127 is in LMC, and its apparent size is much smaller $\left(\simeq 8^{\prime \prime} \times 9^{\prime \prime}\right)$. Both nebulae are quite old (several tens of thousand years). In both cases a preferred axis can be discerned (the major axis of the ellipse) and of two symmetrical regions of enhanced brightness, aligned with the minor axis. While all nebulae in Fig. 1 are relatively young (100-5000 yr) the nebulae in Fig. 2 are ten times older and more, indicating that evolution also plays a role in the formation of the currently observed morphologies.

Kinematical studies of these nebulae have subsequently confirmed what had been previously proposed on the basis of morphological considerations alone. For all known LBVs and Ofpe/WN9 associated nebulae, detailed kinematical studies can be found in the literature.

A Connection between the Large Scale Structure and the Wind Geometry For a number of LBVs, a connection has been found between the large scale structure of the nebulae, up to a few parsecs from the star, and the structure of the stellar wind. In the case of AG Carinae, for example, Leitherer et al. (1994) noted that the stellar spectrum in the optical and the UV wavelengths is dominated by the effects of a massive stellar wind with two components: a slow dense wind, where the bulk of the recombination radiation is emitted, and a faster, less dense wind, visible in the absorption components of the UV P Cygni profiles (with velocities up to $-1000 \mathrm{~km} \mathrm{~s}^{-1}$ ). At the same time, Schulte-Ladbeck et al. (1994) found very large variations of the polarization with time, along a preferred direction which is aligned with the major axis of the optical nebula. These two independent 

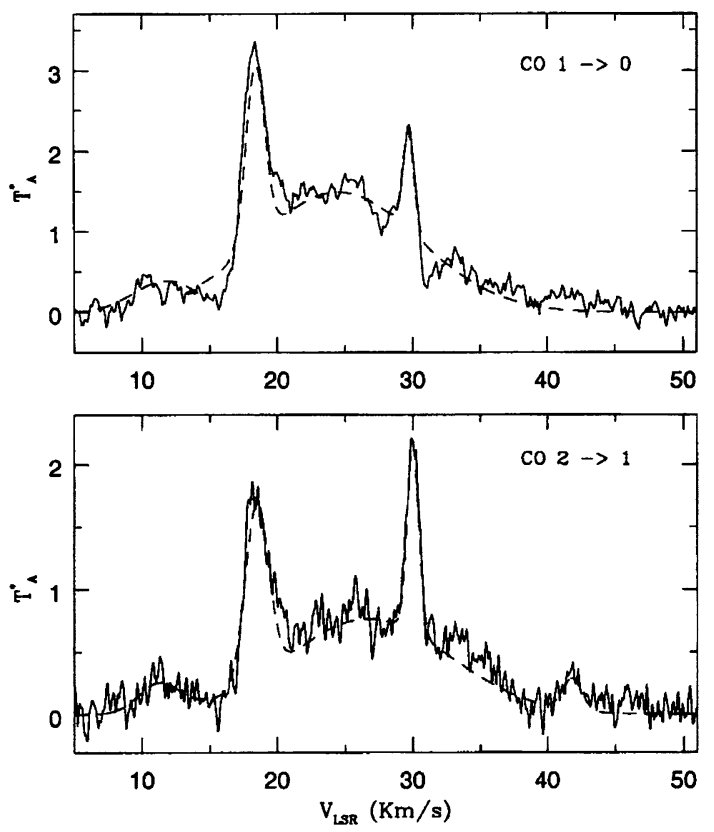

Figure 4. The ${ }^{12} \mathrm{CO} \mathrm{J}=1 \rightarrow 0$ and ${ }^{12} \mathrm{CO} \mathrm{J}=2 \rightarrow 1$ profiles are shown for AG Carinae. Note the broad component indicative of the slow outflow.

observations seem to indicate that the asymmetries noted in the ejected nebula are also present in the stellar wind, very close to the star.

More recently, Nota et at. (2002) published the first detection of ${ }^{12} \mathrm{CO}$ $\mathrm{J}=2 \rightarrow 1$ and ${ }^{12} \mathrm{CO} \mathrm{J}=1 \rightarrow 0$ emission from AG Carinae. They found evidence of a slow outflow of molecular gas, expanding at $\simeq 7 \mathrm{~km} \mathrm{~s}^{-1}$. This emission was unresolved, but spatially localized, rather than extended, and possibly associated with the immediate circumstellar region of AG Carinae. Both detected CO lines were characterized by a pseudo-gaussian profile of $\mathrm{FWHM} \simeq 15 \mathrm{~km} \mathrm{~s}^{-1}$, indicating a slowly expanding region of molecular gas in close proximity to the hot central star. Nota et al. explored possible scenarios to explain the observed profile, and concluded that a circumstellar disk was consistent with additional independent indications for the existence of wind asymmetries in close proximity to the central star, found from spectropolarimetry and analysis of the UV and optical line profiles, and provided the conditions of density and shielding necessary for the survival of the $\mathrm{CO}$ molecules in proximity to such a hot star $\left(\mathrm{T}_{\text {eff }} \simeq 14000 \mathrm{~K}-20000 \mathrm{~K}\right)$. They also derived an estimate of $2.8 \mathrm{M}_{\odot}$ for the total mass of molecular gas, which is smaller, but still comparable with the mass of ionized gas present in the circumstellar environment $\left(4.2 \mathrm{M}_{\odot}\right)$.

Spectropolarimetry of the LMC LBV R127 in UBVRI, $\mathrm{H} \alpha$, [NII] has also shown large intrinsic continuum polarization (1-1.5 \%) indicating both the presence of a substantial quantity of free electrons in the wind and a very large 
deviation from spherical symmetry very close to the star. Again, the direction of the polarization vector matches the nebular symmetry axis (Schulte-Ladbeck et al. 1993) from the optical images.

\subsection{What is Morphology Telling Us}

The prevaling morphologies observed can be explained by different scenarios, such as binarity or interacting winds. Interestingly enough, binarity has been inferred for $\eta$ Carinae to explain the correlation between the spectroscopic variations and the photometric near-infrared light curve. So $\eta$ Car might be the first example of LBV possibly hosting a binary central star. Bipolar morphologies can also be explained by interacting winds. The first inception of this scenario (Nota et al. 1995; Frank, Balick \& Davidson 1995) was derived from the model originally proposed for the shaping of planetary nebulae (Balick 1987; Soker \& Livio 1989; Frank et al. 1993): a spherically symmetrical stellar wind interacts with a pre-existing density contrast between the equatorial and polar directions. As a consequence, the fast wind penetrates more easily in the polar direction, generating two bipolar "bubbles", while a narrower "waist" is compressed in the equatorial direction. Depending on whether the density contrast is large or small, a pronounced bipolar or elliptical morphology is generated, respectively. Several variations of the basic model have been proposed in the recent years, ranging from the model proposed by Frank, Ryu \& Davidson 1988 and Dwarkadas \& Balick 1988 based on an aspherical fast wind expanding into a slow, spherical wind, to the comprehensive approach of Langer, Garcia-Segura \& McLow (1999), who use the wind compressed disc model by Cassinelli \& Bjorkman (1993) to tie the nebular structure to its formation and to the evolution of the central star. In their proposed scenario, at the moment of the LBV outbursts, which occur when the star has reached critical rotation (its Eddington limit), a slow dense equatorial wind is followed by a fast, almost spherical wind.

Alternatively, stellar rotation can produce strongly anisotropic mass loss. It was first noted by Owocki \& Gayley (1997) that in a star rotating near the critical Omega limit the equatorial gravity darkening of the stellar radiation field can lead naturally to a bipolar wind outflow. Recently, this concept had been further developed by Meader \& Desjacques (2001), Maeder (2002), and Pelupessy et al. (2002), to the conclusion that stellar winds from rotating stars show two main components: a fast, low density wind, produced by the higher $\mathrm{T}_{\text {eff }}$ and gravity at the poles, and an equatorial disc with a slow dense wind, produced by the stronger opacities at the equator. Such a scenario well explains the wind asymmetries found for many LBVs in the UV and from spectropolarimetry, but also produces models that morphologically well match the observations. In Fig. 3 , we show the comparison between a simulation from Meader \& Desjacques (2001) with $T_{\text {eff }} \simeq 30000 \mathrm{~K}$ which generates an elliptical nebula, here compared with the LBV nebula around AG Carinae (upper panel). We also show that at lower temperatures $\left(\mathrm{T}_{\text {eff }}<25000 \mathrm{~K}\right)$, the presence of a bi-stability limit at the equator generates an equatorial disc, such as in the case of $\eta$ Carinae (lower panel). 

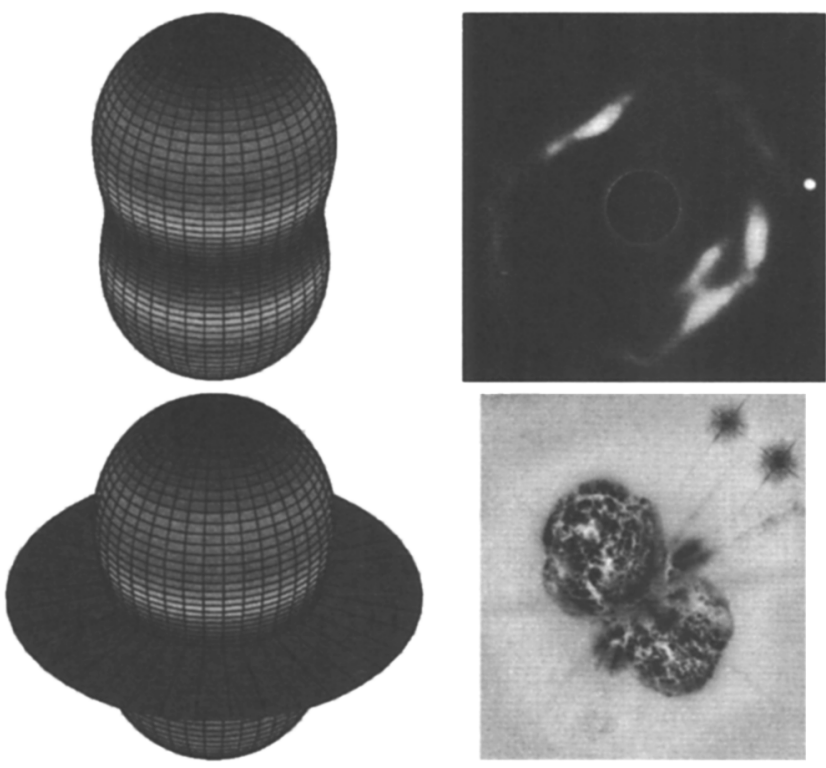

Figure 5. A comparison between the elliptical nebula simulated by Meader $\&$ Desjacques (2001) at $\mathrm{T}_{\text {eff }} \simeq 30000 \mathrm{~K}$ and the nebula around te LBV AG Carinae (uper panel). At lower temperatures $\left(\mathrm{T}_{\text {eff }}<25000 \mathrm{~K}\right)$, the presence of a bi-stability limit at the equator generates an equatorial disc, and the model is here compared with $\eta$ Carinae (lower panel).

\subsection{The Chemical Composition}

Chemical abundances have been determined for a significant number of LBV and Ofpe/WN9 nebulae (see Table 2).

Table 2. N/O ratios for nebulae around LBVs and Ofpe/WN9 stars

\begin{tabular}{lll}
\hline Name & N/O & Reference \\
\hline$\eta$ Car $^{(*)}$ & & Dufour 1997 \\
P Cygni $^{(*)}$ & $>0.16$ & Johnson et al. 1992 \\
AG Carinae $^{(*)}$ & $6 \pm 2$ & Mitra \& Dufour 1990; Smith et al. 1997 \\
S119 & $1.9 \pm 0.5$ & Smith et al. 1998 \\
R127 & $0.9 \pm 0.4$ & Smith et al. 1998 \\
\hline
\end{tabular}

In general, $\mathrm{N} / \mathrm{O}$ ratios have been found higher than initial. With the exception of $\eta$ Carinae, $\mathrm{N}$ and $\mathrm{O}$ abundances are found smaller that expected for gas with CNO-equilibrium abundances (Lamers et al. 2001). Let's take for example the case of R127, where chemical abundances have been quite accurately determined from HST spectra (which could minimize the stellar contamination). For $\mathrm{R} 127, \log \mathrm{N} / \mathrm{H}=8.05 \pm 0.08$, and $\log \mathrm{O} / \mathrm{H}=8.10 \pm 0.17$, compared with LMC HII abundances of 7.02 and 8.40 respectively. $\mathrm{N}$ results to be overabundant by 
a factor 10.7 , and $\mathrm{O}$ is depleted by a factor 2 . CNO equilibrium abundances predict $\mathrm{N}$ overabundance by 13 , and $\mathrm{O}$ depletion by 18 . The values measured instead are consistent with the convective envelope of a Red Supergiant RSG).

Stars more massive than $40 \mathrm{M}_{i}$ are not expected to become RSGs. Stellar rotation can explain the discrepancy. Lamers et al. (2001) have calculated the changes in surface composition during the main sequence phase due to rotation induced mixing (Heger \& Langer 2000; Meynet \& Maeder 2000). They found that the predicted $\mathrm{He} / \mathrm{H}$ ratios in the nebulae are significantly smaller than the observed photospheric values of their central star at present. They concluded that LBV nebulae are ejected during the BSG phase and the chemical enrichment is due to rotation induced mixing while the ejection is possibly triggered by nearcritical rotation.

Additional evidence is provided by the study of Sher 25, a blue supergiant $\left(\mathrm{M} \simeq 60 \mathrm{M}_{\odot}\right.$ ) in NGC3603 (Smartt et al. 2002) Sher 25 is surrounded by a spectacular nebula which includes an equatorial ring and probable bipolar outflows. Brandner et al. (1997) posed the question on whether the nebula had been ejected during a previous RSG phase. Smartt et al. (2002) performed a detailed chemical analysis of Sher 25 and found that $\mathrm{N} / \mathrm{O} \simeq 0.36$, a value that is much lower than what Lamers et al. (2001) had predicted for convective dredge up in a RSG phase. However, CNO abundances suggest some $\mathrm{CN}$ and NO processed gas mixing, consistent with some form of rotationally induced mixing during or after the MS phase.

It will be very interesting to study the chemical abundances of the nebula and compare the findings with the surface composition of the star.

\section{Conclusion}

I have shown in this review that nebulae around evolved massive stars do provide a wealth of information that can be used to improve our understanding of the latest stages of massive star evolution. I have shown that nebular morphology and chemical abundances provide independent support that stellar rotation has played a major role in the mai sequence and post main sequence evolution for these stars.

\section{References}

Barlow, M.J., Drew,J.E., Meaburn,J. \& Massey, R.M. 1994, MNRAS 268, L29

Clampin, M., Nota, A., Golimowski, D.A., Leitherer, C. \& Durrance, S.T. 1993, ApJ 410, L35

Clampin, M., Schulte-Ladbeck, R.E., Nota, A., Robberto, M., Paresce, F., \& Clayton, G. 1995 AJ 110, 251

Davidson, K., Ebbets, D., Johansson, S., Morse, J.A., Hamann, F.W., Balick, B., Humphreys, R., Weigelt, G. \& Frank, A. 1997, AJ 113, 335

Damineli, A. 1996, ApJ 460, L49

de Winter, D., Peréz, M.R., Hu, J.H. \& Thé, P.S. 1992, A\&A 257, 632

Frank, A., Balick, B. \& Davidson, K. 1995, AJ 109, 178

García-Segura,G., Langer, N. \& MacLow, M.,-M. 1996, A\&A 316, 133 
Grosdidier, Y. Moffat, A.F.J., Joncas, G. \& Acker, A. 1998, ApJ 506, L127

Hu, J.H., de Winter, D., Thé, P.S. \& Peréz, M.R. 1990, A\&A 227, L17

Hulbert, S., Nota, A., Clampin, M., Leitherer, C., Pasquali, A., Langer, N. \& SchulteLadbeck, R.E. 1998, this conference

Hutsemékers, D. \& Van Drom, E. 1991a A\&A 248, 141

Hutsemékers, D. \& Van Drom, E. 1991b, A\&A 251, 620

Hutsemékers, D., Van Drom, E., Gosset, E. \& Melnick, J. 1994 A\&A 290, 906

Leitherer, C., Allen, R., Altner, B., Damineli, A., Drissen, L., Idiart, T., Lupie, O., Nota, A., Robert, C., Schmutz, W. \& Shore, S.N. 1994, ApJ 428, 292

Nota, A., Livio, M., Clampin, M. \& Schulte-Ladbeck, R.E. 1995, ApJ 448, 788

Nota, A., Pasquali, A., Clampin, M., Pollacco, D., Scuderi, S. \& Livio, M. 1996 ApJ 473, 946

Nota, A. \& Clampin, M. 1997: Luminous Blue Variables: Massive Stars in Transition eds. A.Nota, H.J.L.M. Lamers (PASP), p.303

Nota, A., Smith, L.J., Pasquali, A., Clampin, M., \& Stroud, M. 1997 ApJ 486, 338

Nota, A., Clampin, M., Pasquali, A., Robberto, M., Pollacco, D., Ligori, S., Paresce, F. \& Staude, J. 1998 in preparation.

Parker, J.Wm., Clayton, G.C., Winge, C. \& Conti, P. 1993, ApJ 409, 770

Robberto, M., \& Herbst, T.M. 1998, ApJ 498, 400

Schulte-Ladbeck, R.E., Leitherer, C., Clayton, G.C., Robert, C., Meade, M.R., Drissen, L. Nota, A. \& Schmutz, W. 1993, ApJ 407, 723

Schulte-Ladbeck, R.E., Clayton, G.C., Hillier, D.J., Harries, T.J. \& Howarth, I.D. 1994, ApJ 429, 846

Schulte-Ladbeck, R.E., Pasquali, A., Clampin, M., Nota, A., Hillier, D.J.\& Lupie, O. 1998, this conference.

Sirianni, M., Nota, A., Pasquali, A. \& Clampin, M. 1998, A\&A 335, 1029

Skinner, C., Becker, R.H., White, R.L., Exter, K.M., Barlow, M.J. \& Davis, R.J. 1998, MNRAS 296, 669

Smith, L.J., Stroud, M.P., Esteban, C., Vilchez, J.M. 1997, MNRAS 290, 265

Smith, L.J., Nota, A., Pasquali, A., Leitherer, C., Clampin, M. \& Crowther, P.A. 1998 ApJ 503, 278

Stahl, O. 1987 A\&A 182, 229

Taylor, M., Nordsieck, K.H., Schulte-Ladbeck, R.E. \& Bjorkman, K.S. 1991 AJ 102, 1197

Trams, N.R., Waters, L.B.F.M. \& Voors, R.H.M. 1996, A\&A 315, L213

Walborn, N.R. 1982, ApJ 256, 452

Weis, K., Duschl, W.L., Bomans, D.J., Chu, Y.-H., \& Joner, M.D. 1997 A\&A 320, 568 